\title{
Spatiotemporal toroidal waves from the transverse second-harmonic generation
}

\author{
Solomon M. Saltiel ${ }^{1,2}$, Dragomir N. Neshev ${ }^{1}$, Robert Fischer ${ }^{1}$, \\ Wieslaw Krolikowski ${ }^{1}$, Ady Arie $^{3}$, and Yuri S. Kivshar ${ }^{1}$ \\ ${ }^{1}$ Nonlinear Physics Center and Laser Physics Center, \\ Center for Ultra-high bandwidth Devices for Optical Systems (CUDOS), \\ Research School of Physical Sciences and Engineering, \\ Australian National University, Canberra ACT 0200, Australia \\ ${ }^{2}$ Department of Quantum Electronics, Faculty of Physics, Sofia University, Bulgaria \\ ${ }^{3}$ School of Electrical Engineering, Faculty of Engineering, Tel-Aviv University, Tel-Aviv, Israel
}

We study the second-harmonic generation via transversely-matched interaction of two counter-propagating ultra-short pulses in $\chi^{(2)}$ photonic structures. We show that the emitted second-harmonic wave attains the form of spatially expanding toroid with the initial thickness given by the cross-correlation of the pulses. We demonstrate the formation of such toroidal waves in crystals with random ferroelectric domains as well as in annularly poled nonlinear photonic structures.

(C) 2007 Optical Society of America

OCIS codes: $190.0190,190.4420,260.5950$

Transverse second-harmonic generation (TSHG) is a challenging nonlinear optical effect $[1,2]$ that can not be observed in bulk homogeneous nonlinear crystals since the phase matching condition cannot be satisfied. So far, the TSHG has been observed mostly in thin $(1-2 \mu \mathrm{m}$ thick) quantum-well waveguide structures [3-6]. Noncollinear second harmonic $(\mathrm{SH})$ generation with waves emitted in all directions of a plane that contains the fundamental wave, was also recently demonstrated with beams propagating along the $\mathrm{X}$ or $\mathrm{Y}$ crystalline axis of randomly poled nonlinear crystals $[7,8]$. However, if the single fundamental beam (pulse) propagates along the Z-axis, the TSHG phase-matching conditions cannot be fulfilled.

In this Letter, we report on the observation of volume pure TSHG in a plane perpendicular to the Z-directed fundamental beams. This is achieved by interaction of two counter-propagating femtosecond pulses along the optical axis of a quadratic nonlinear crystal. We observe the second harmonic ( $\mathrm{SH}$ ) generated in the form of an expanding toroidal wave (T-wave) emitted from the overlap zone of the counter-propagating pulses.

Ferroelectric crystals with alternating sign of second order nonlinearity provide effective means to quasi-phasematch nonlinear processes such as frequency doubling. While typically the light propagates in the crystallographic X-Y plane, in our experiments we use fundamental beams directed along the optical Z-axis [Fig. 1(a)]. We use two different types of samples with $\chi^{(2)}$ spatial modulation: a $5 \mathrm{~mm}$-thick Strontium Barium Niobate (SBN) crystal with naturally disordered domain structure [9] and an annular periodically poled Stoichiometric Lithium Tantalate (SLT) [10]. The SLT sample is $0.49 \mathrm{~mm}$ thick and the poling period is $7.5 \mu \mathrm{m}$ [Fig. 1(f)].

In contrast to the fixed-period SLT structure, random distribution of anti-parallel ferroelectric domains of an "as-grown" SBN leads to a natural disordered photonic structure [Fig.1(d)]. This structural disorder provides almost a continuous set of grating vectors in the $\mathrm{X}-\mathrm{Y}$ plane of the crystal allowing to phase-match parametric processes over a broad range of frequencies [11, 12].

As we already noted no TSHG cannot take place for the fundamental beam propagating along the Z-axis. Instead, each pulse emits continuously a SH signal in the form of a cone [7, 12], as shown schematically by the cones on the both side of the crystal in Fig. 1(a). These cones are clearly seen in the experiments with SBN as two strong outer lines as shown in Fig. 1(c). Analogous conical emission takes place in the annular periodically poled sample of SLT [see two side lines in Fig. 1(e)]. The two cones are formed independently of the alignment of the two oppositely directed beams. Only when the two beams are exactly overlapped transverse second harmonic wave is radiated. In such case the momenta of the two counter-propagating photons cancel out, and the transverse phase-matching can be achieved due to the reciprocal grating vector provided by the nonlinearity modulation. This can be seen clearly from the phase-matching diagram shown in the inset of Fig. 1(a). The solid arrow represents the effective reciprocal lattice vectors $\mathbf{g}$ while the dotted (red) and the dashed (blue) arrows denote the fundamental $\mathbf{k}_{\omega}$ and $\mathrm{SH} \mathbf{k}_{\mathbf{2} \omega}$ wavevectors, respectively. Due to this phase-matching restrictions the $\mathrm{SH}$ is emitted only from the region of pulse overlap and only for the duration of the pulse interaction. It is exactly this spatiotemporal overlap that allows for the emission of a spatiotemporal wave of a toroidal shape [illustrated by the central ring in Fig. 1(a)]. The width and intensity profile of this wave along $\mathrm{Z}$ direction is determined solely by the temporal correlation of the fundamental pulses, while the width and intensity profile in the transverse $(\mathrm{X}-\mathrm{Y})$ direction (propagation direction of the 

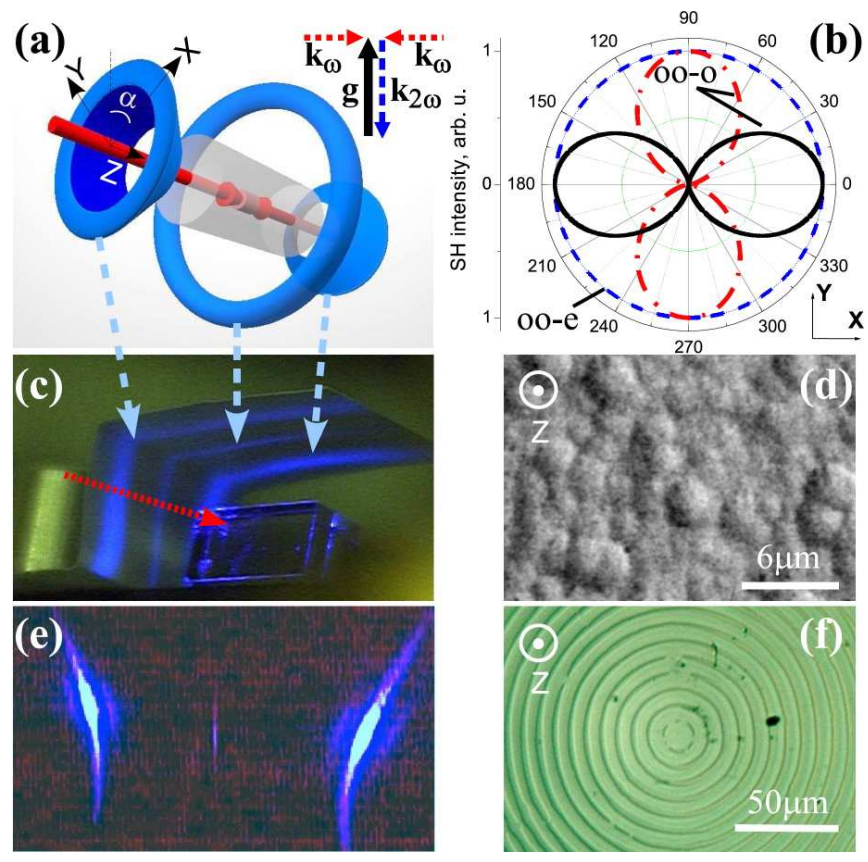

(1)

Fig. 1. (color online) (a) Generation of SH T-waves with two counter-propagating beams. Inset: phase-matching diagram. (b) Theoretically determined emission diagram in polar coordinates $\left(I_{2}, \alpha\right)$ of a T-wave: Dashed line phase matched OO-E interactions (in SLT and SBN); Dashed-doted and solid lines: OO-O interaction (in SLT only) for both input polarizations either parallel along $\mathrm{X}$, or $\mathrm{Y}$ (dash-dotted) or mutually perpendicular along to $\mathrm{X}$ and Y directions (solid). (c) SH signal (emitted from $\mathrm{SBN}$ ) as seen on the rectangular screen around the crystal. The two outer traces are the conical waves and the weaker central line represents the T-wave. (d) Domain structure in "as grown" SBN sample [9]. (e) Experimental image of a T-wave (central trace) and conical waves generated in the SLT sample; (f) photo of the annular periodic domain pattern in SLT.

T-wave) depends on pulse length and the fundamental beams spatial profiles.

In our experiments we use pulses from a regenerative Ti:Sapphire amplifier operating at a wavelength of $830 \mathrm{~nm}$. The system delivers linearly polarized $165 \mathrm{fs}$ long pulses of energy up to $3 \mu \mathrm{J}$ at a repetition rate of $250 \mathrm{kHz}$. The beam with a Gaussian spatial profile is split in a polarizing beam splitter and directed from both sides to a quadratic nonlinear medium such that the same pulses meet roughly in the center of the sample. A set of $\lambda / 2$ plates allows to control the relative powers of both beams and their polarizations. The average beam power before the beam-splitter is $\sim 340 \mathrm{~mW}$. The two beams are loosely focused in the sample to $160 \mu \mathrm{m}$ waist. All facets of both samples are polished and the emitted $\mathrm{SH}$ signal is recorded by a CCD camera.

The first-order TSHG phase-matching requires very fine grating periods. For the SLT sample, the necessary period is $183 \mathrm{~nm}$ for a $830 \mathrm{~nm}$ fundamental wave. As the grating period of our SLT sample is $7.5 \mu \mathrm{m}$ the observed TSHG is thus due to a 41-st order phase-matching which, to the best of our knowledge, is the highest QPM order in crystals reported so far [1]. Naturally, such a high-order process results in a very low efficiency. The quadratic dependence of the SH signal on the power of the fundamental wave is verified by measuring the $\mathrm{SH}$ intensity in a particular single direction. To obtain angularly symmetric TSHG, it is essential to focus both counter-propagating beams exactly at the center of the annular domain structure. In contrast, the TSHG in SBN does not depend critically on the position of the beams in the sample since the phase-matching conditions are the same everywhere in the crystal. Furthermore, since the average domain size is approximately $2.5 \mu \mathrm{m}$, the phase matching order is 14 , resulting in higher second harmonic generation efficiency.

As different nonzero values of the $\chi^{(2)}$ components are involved in TSHG in SBN and SLT, the emission diagrams for both structures are also different. The calculated emission diagrams in polar coordinates $\left(I_{2}, \alpha\right)$ where $\alpha$ is the emission (observation) angle for the ordinary and extraordinary polarized T-waves are shown in Fig. 1(b). In the case of the OO-E interaction (ordinary polarized fundamental beams and extraordinary polarized $\mathrm{SH}$ ) the relevant nonzero $\chi^{(2)}$ components in both crystals are $d_{z x x}$ and $d_{z y y}=d_{z x x}$. The generated $\mathrm{T}$-wave is polarized along the Z-axis of the crystal and its intensity is constant for all emission directions in the $\mathrm{X}-\mathrm{Y}$ plane. However, the $\mathrm{SH}$ intensity depends critically on the polarization of the fundamental waves $I_{2, \text { ex }} \propto\left[d_{z y y} I_{1} \cos \left(\gamma_{1}-\gamma_{2}\right)\right]^{2}$, where $\gamma_{1}, \gamma_{2}$ denote the angles of input polarizations for both the beams measured counterclockwise with respect to the $\mathrm{X}$-axis. In contrast to SBN where the OO-O interaction is impossible, in SLT the relevant $\chi^{(2)}$ components $d_{y y y}$ and $d_{y x x}=-d_{y y y}$ allow for the generation of an ordinary $\mathrm{SH}$ with its intensity varying with the emission angle $\alpha$ and input polarization directions as $I_{2, o} \propto\left[d_{\text {yyy }} I_{1} \sin \left(\gamma_{1}+\gamma_{2}-\alpha\right)\right]^{2}$.

Experimental emission traces are shown in Fig. 1(c) for SBN $(0 \leq \alpha \leq \pi)$ and in Fig. 1(e) for SLT, $(\alpha=\pi)$. However, since our samples are not cylindrical, it was not possible to measure accurately the angular variations of the intensity of $\mathrm{SH}$. Instead, we measured the $\mathrm{SH}$ intensity and polarization properties of the emitted wave along the $\mathrm{X}$ - and $\mathrm{Y}$-axis of the crystal vs. the polarization angles $\gamma_{1}, \gamma_{2}$. The experimental results for both SBN and SLT crystals are shown in Fig. 2 together with the curves representing theoretical fit. The graphs in Fig. 2(a) show the measured $\mathrm{SH}$ signal emitted in the SBN crystal (points) as a function of $\gamma_{1}$ for few values of $\gamma_{2}$. The agreement with the expression for $I_{2, e}$ (solid lines) of the OO-E interaction is excellent. For the SLT crystal the polarization dependencies are more complicated due to the simultaneous contribution of both OO-O and OO-E interactions [see Figs. 2(b,c)]. Fig. 2 (b) shows the dependence of the total intensity of the $\mathrm{SH}$ signal on 


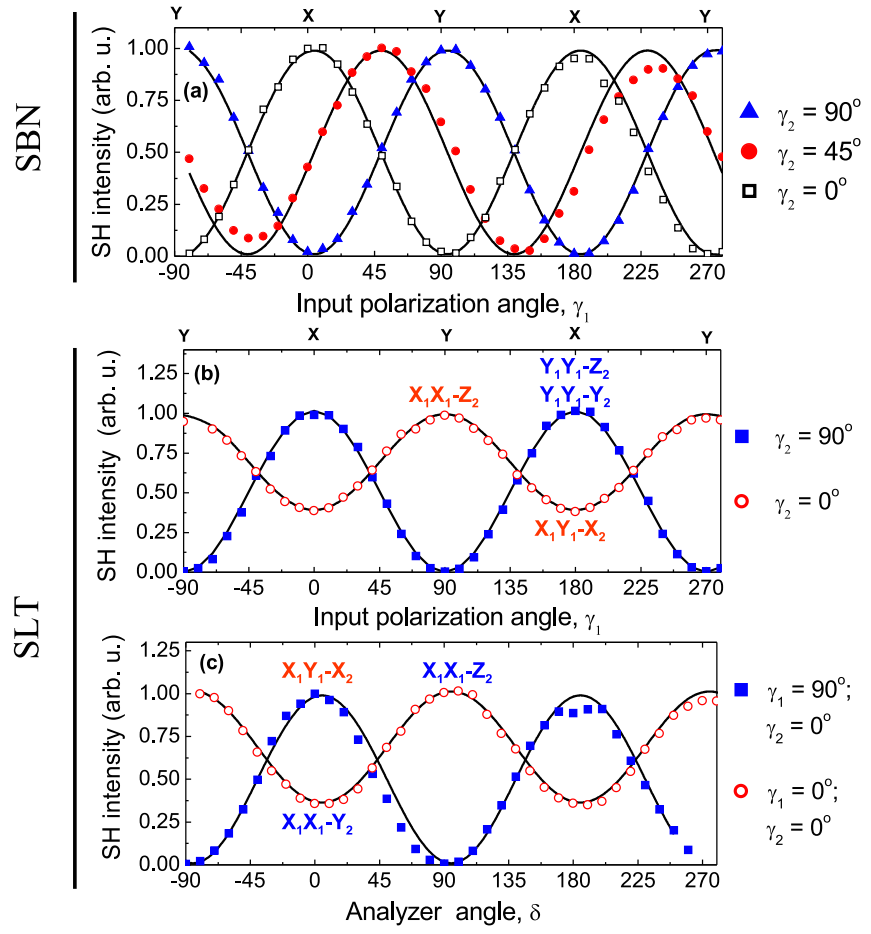

Fig. 2. (color online) Polarization characteristics of SHG in (a) SBN and (b-c) SLT: (a) Intensity of the SH signal as a function of the input polarization angle $\gamma_{1}$, for three values of $\gamma_{2}$; (b) total SH signal in the SLT sample as a function of the input polarization angle $\gamma_{1}$ for indicated values of $\gamma_{2}$; (c) Intensity of the SH signal generated in the SLT sample as a function of the angle $(\delta)$ of an analyzer mounted in front of the CCD camera. In all plots solid lines represent the theoretical fits.

the polarization of the fundamental beams. On the other hand, in the case displayed in Fig. 2(c) both fundamental beams were either parallel (X direction - circles) or orthogonally (X and Y directions - squares) polarized. The SH signal is then measured as a function of the angular position $(\delta)$ of the analyzer mounted in front of the CCD camera. For parallel polarized fundamental beams, both OO-E and OO-O processes contribute to the $\mathrm{SH}$ signal. Hence the recorded signal contains both ordinary and extraordinary components and never vanishes. For orthogonally polarized input beams, the $\mathrm{SH}$ wave is ordinary polarized (due to OE-O process) and the recorded $\mathrm{SH}$ signal vanishes at the angles $\delta=(-\pi / 2, \pi / 2,3 \pi / 2)$.

A quantitative analysis of the experimental data indicates that the contribution of the OO-E process governed by the $d_{z y y}$ component is stronger than that of the OO$\mathrm{O}$ process governed by the $d_{y y y}$ component. This does not agree with the value $d_{z y y} / d_{y y y}=0.59$ reported earlier [13]. This contradiction can be explained by the fact that OO-E process is closer to the exact phase matching condition than the OO-O process. We also note that for the SBN sample the SH generation process is practically independent of the fundamental wavelength within a broad frequency range.

Due to the transverse geometry of the parametric interaction, the TSHG signal effectively translates the time coordinate into the space coordinate such that the width of the $\mathrm{T}$-wave in the direction of the $\mathrm{Z}$-axis is exactly the autocorrelation function of the interacting pulses $[3,4,8]$. From calibrated experimental photo similar to one in Fig.1(e) we measured the thickness of the toroid wave in SLT crystal to be $34 \mu \mathrm{m}$, that corresponds to $160 \mathrm{fs}$ assuming secant hyperbolic temporal shape. Since the beam size is much bigger than the spatial extent of the pulse, the thickness of the T-waves in propagation direction outside the sample is determined by the beam size and is about $250 \mu \mathrm{m}$

In conclusion, we have generated toroidal secondharmonic waves via interaction of counter-propagating femtosecond pulses in annularly poled SLT structures and SBN crystals with disordered domains. As the thickness of the T-wave is determined by the correlation function of the fundamental pulses, this effect can be used as a background-free single short-pulse autocorrelator.

This work was supported by the Australian Research Council and the Israeli Science Foundation (grant no. 960/05). We thank D. Kasimov, A. Bruner, P. Shaier, and D. Eger for the SLT sample, and Jose Garcia Solé for a photo of the SBN domain structure.

\section{References}

1. X. Gu, R. Y. Korotkov, Y. J. Ding, J. U. Kang, and J. B. Khurgin, J. Opt. Soc. Am. B 15, 1561 (1998).

2. G. D. Landry and T. A. Maldonado, J. Opt. Soc. Am. B 21, 1509 (2004).

3. R. Normandin, R. L. Williams, and F. Chatenoud, Electron. Lett. 26, 2088 (1990).

4. N. D. Whitbread, J. A. R. Williams, J. S. Roberts, I. Bennion, and P. N. Robson, Opt. Lett. 19, 2089 (1994).

5. A. Fiore, Y. Beaulieu, S. Janz, J. P. McCaffrey, Z. R. Wasilewski, and D. X. Xu, Appl. Phys. Lett. 75, 2655 (1997).

6. T. M. Crawford, C. T. Rogers, T. J. Silva, and Y. K. Kim, Appl. Phys. Lett. 68, 1573 (1996).

7. A. R. Tunyagi, M. Ulex, and K. Betzler, Phys. Rev. Lett. 90, 243901 (2003).

8. R. Fischer, D. N. Neshev, S. M. Saltiel, A. A. Sukhorukov, W. Krolikowski, and Yu. S. Kivshar, Appl. Phys. Lett. 91, 031104 (2007).

9. J. J. Romero, D. Jaque, J. Garca Solé, and A. A. Kaminskii Appl. Phys. Lett. 78, 1961 (2001).

10. D. Kasimov, A. Arie, E. Winebrand, G. Rosenman, A. Bruner, P. Shaier, and D. Eger, Opt. Exp. 14, 9371 (2006).

11. J. J. Romero, C. Arago, J. A. Gonzalo, D. Jaque, and J. Garcia Solé, J. Appl. Phys. 93, 3111 (2003).

12. R. Fischer, S. M. Saltiel, D. N. Neshev, W. Krolikowski, and Yu. S. Kivshar, Appl. Phys. Lett. 89, 19110 (2006). 
13. F. Charra and G. G. Gurzadyan, in: Nonlinear Dielectric Susceptibilities, ed. D. F. Nelson (Springer,
Berlin, 2000). 